\title{
Detrended fluctuation analysis of earthquake data
}

\author{
Takumi Kataoka $\odot,{ }^{1}$ Tomoshige Miyaguchi $\odot,{ }^{2}$ and Takuma Akimoto $\odot^{1, *}$ \\ ${ }^{1}$ Department of Physics, Tokyo University of Science, Noda, Chiba 278-8510, Japan \\ ${ }^{2}$ Department of Mathematics, Naruto University of Education, Naruto, Tokushima 772-8502, Japan
}

(Received 19 April 2021; accepted 4 June 2021; published 22 July 2021)

\begin{abstract}
The detrended fluctuation analysis (DFA) is extensively useful in stochastic processes to unveil the long-term correlation. Here, we apply the DFA to point processes that mimic earthquake data. The point processes are synthesized by a model similar to the epidemic-type aftershock sequence model, and we apply the DFA to time series $N(t)$ of the point processes, where $N(t)$ is the cumulative number of events up to time $t$. Crossover phenomena are found in the DFA for these time series, and extensive numerical simulations suggest that the crossover phenomena are signatures of nonstationarity in the time series. We also find that the crossover time represents a characteristic time scale of the nonstationary process embedded in the time series. Therefore, the DFA for point processes is especially useful in extracting information of nonstationary processes when time series are superpositions of stationary and nonstationary signals. Furthermore, we apply the DFA to the cumulative number $N(t)$ of real earthquakes in Japan, and we find a crossover phenomenon similar to that found for the synthesized data.
\end{abstract}

DOI: 10.1103/PhysRevResearch.3.033081

\section{INTRODUCTION}

Although stationarity is one of the most important properties in theoretical foundations of stochastic processes, nonstationary phenomena are rather ubiquitous in nature, ranging from disordered systems [1-6], to seismicity [7-10], to biological systems [11-13]. Therefore, in general, one cannot assume stationarity in real data analysis. Particularly in point processes, there are two typical types of nonstationary processes. The first (nonstationarity of the first type) is a process in which the probability density function (PDF) for recurrence times depends explicitly on time. Typical examples are rainfalls that exhibit daily and seasonal alterations. The other (nonstationarity of the second type) is a process where a characteristic time scale of the process, such as the mean of the interval between consecutive points, diverges. Owing to divergence, the process never reaches a steady state and thus exhibits nonstationary behaviors $[2,6,14]$. In this study, we focus on nonstationary processes of the first type.

Earthquakes are an example of the nonstationary point process of the first type. One of the most well-known statistical laws of seismicity is the Gutenberg-Richter law [15], which states that the magnitude distribution of earthquakes follows an exponential distribution. This statistical law is universal in the sense that the exponential distributions are observed in any region on earth, any periods, and any types

\footnotetext{
*takuma@rs.tus.ac.jp

Published by the American Physical Society under the terms of the Creative Commons Attribution 4.0 International license. Further distribution of this work must maintain attribution to the author(s) and the published article's title, journal citation, and DOI.
}

of earthquakes such as mainshocks and aftershocks. However, the decay constant, the so-called $b$ value, depends on time [16]; thus, it is a nonstationary law. Additionally, the Omori law describes a nonstationary property for aftershocks [7], which states that the occurrence rate of aftershocks decays with the time elapsed from the mainshock. More precisely, the occurrence rate decays as a power law: $\lambda(t) \propto t^{-p}$ for large $t$, where $\lambda(t)$ is the occurrence rate at elapsed time $t$ after a mainshock, and $p(>0)$ is a parameter. Since the rate of aftershocks, $\lambda(t)$, explicitly depends on $t$, aftershocks are intrinsically nonstationary, and earthquake occurrences are nonstationary processes of the first type.

Several methods are proposed to analyze nonstationary time series. For diffusion in heterogeneous environments, trajectories of a diffusing particle can be tracked, and using the trajectory data, the diffusion coefficient can be obtained from the time-averaged mean square displacement (MSD) calculated from the trajectory. If the process is nonstationary, the diffusion coefficient depends explicitly on the total measurement time $[6,11,12,17-20]$. Thus, plotting the diffusion coefficient as a function of the measurement time provides us information on how the process ages. In another method of nonstationary data analysis of the second type, the interoccurrence times are utilized frequently $[11,21,22]$. For example, the interoccurrence-time PDFs have also been extensively used for earthquake research [23-27].

Although the time-averaged MSD and interoccurrencetime PDF are useful analysis methods for nonstationary time series of the second type, these methods are not effective in the case of the first type. In nonstationary processes of the first type, a long-measurement-time limit of a time average may have a definite value, as the process does not age monotonically. Thus, the diffusion coefficient of time-averaged MSD does not depend on the total measurement time. Moreover, 
the interoccurrence-time PDF analysis is based on the fact that the interoccurrence times are independent and identically distributed (IID). However, this assumption is not valid for nonstationary processes of the first type. Therefore, it is important to develop a method to extract information of nonstationary features from the time series.

In this study, we utilize the detrended fluctuation analysis (DFA) to overcome the above-mentioned difficulties of nonstationary time-series analysis of earthquakes [28]. The difference between the DFA and the time-averaged MSD is that the local trends are subtracted in the DFA. The DFA of recurrence times of earthquakes in stationary regimes was studied to unveil the long-term correlation [29]. We note that the DFA can be utilized irrespective of the interoccurrence times being IID. Here, we apply the DFA to both data synthesized by an earthquake model and data of real earthquakes in nonstationary regimes. In particular, we perform the DFA on a cumulative number $N(t)$ of earthquakes that occurred up to time $t$. As an earthquake model, we employ a simplified version of the epidemic-type aftershock sequence model [9].

We find crossover phenomena in DFA for both synthesized and real earthquake data. It is shown that the crossover time represents a characteristic time scale of a nonstationary process. Moreover, we present analytical predictions of long-time behaviors in DFA for point processes. Until now, the DFA for fractional Brownian motion (FBM) was studied in Ref. [30], and, more recently, relations between the DFA, autocorrelation functions, and power spectral densities have been analytically obtained [31-33]. In this paper, we obtain an analytical expression for point processes. Because point processes are different stochastic processes from the FBM [34], theoretical foundations of the DFA for point processes are important. The DFA has been widely used in data analysis, and thus the analytical results for point processes are also useful.

This article is organized as follows. In Sec. II, the earthquake model, which is a superposition of stationary and nonstationary point processes, is proposed, and in Sec. III, the DFA method is briefly reviewed. Sections IV and V present results of DFA for synthesized data and real earthquake data, respectively. Finally, Sec. VI presents a summary and discussion.

\section{EARTHQUAKE MODEL}

Here, we propose a point process describing occurrences of earthquakes over a vast area, such as the entire extent of Japan. In our model, three types of earthquakes are considered: mainshocks, aftershocks, and stationary earthquakes independent of mainshocks and aftershocks (background earthquakes).

First, we assume that mainshocks occur independently, and thus they are described by a Poisson process. This assumption is quite reasonable because the superposition of large numbers of mutually independent renewal processes becomes a Poisson process in general [35]. Moreover, there are studies that state that mainshock occurrences have been considered a Poisson process $[36,37]$. We have partially confirmed this assumption for a region around Japan by analyzing the Japan Meteorological Agency (JMA) catalog [38] where we define the mainshocks as earthquakes with magnitudes greater than 7 .

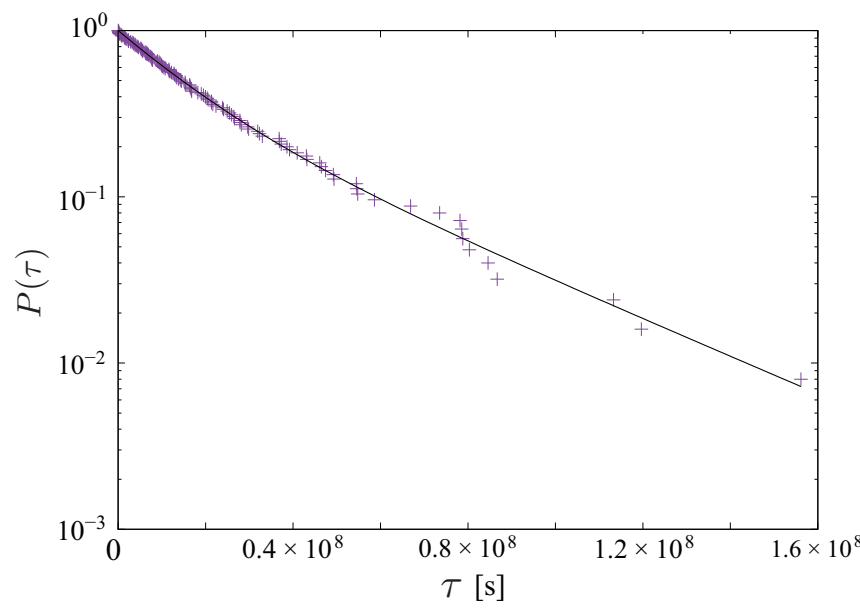

FIG. 1. Survival probability $P(\tau)$ of interoccurrence times of mainshocks (semilogarithmic plot). There have been 135 mainshocks from 1 January 1923 to 30 April 2017 in Japan. Crosses are a result of real earthquake data. The solid line represents a superposition of the exponential distributions, i.e., $P(\tau)=A \exp \left(-\lambda_{1} \tau\right)+$ $(1-A) \exp \left(-\lambda_{2} \tau\right)$, with $A=0.59, \lambda_{1}^{-1} \cong 1.48 \times 10^{7} \mathrm{~s}$, and $\lambda_{2}^{-1} \cong$ $3.87 \times 10^{7} \mathrm{~s}$.

Figure 1 shows that survival probability $P(\tau)$ of interoccurrence times $\tau$ between successive mainshocks follows a superposition of exponential distributions. In the data, the mean interoccurrence time is around $2.20 \times 10^{7} \mathrm{~s} \cong 254 \mathrm{~d}$. The exponential distribution with mean $2.20 \times 10^{7} \mathrm{~s}$ also well describes the interoccurrence distribution. Therefore, the mainshock rate $\lambda_{m}$ for the entire extent of Japan is approximately given by $\lambda_{m} \cong 1 / 2.2 \times 10^{-7} 1 / \mathrm{s}$. We note that it not a solid conjecture [39]. However, in this paper, this assumption is not important and is used only in Sec. IV C. Thus, the assumption on mainshock occurrences does not affect our main findings. In other words, results except for Sec. IV C do not depend on this assumption.

Second, we assume that aftershocks are triggered by a mainshock. In particular, we assume the Omori law [7], which states that the rate of occurrence of aftershocks after a mainshock follows a power-law decay:

$$
\lambda_{a}(t)=\frac{K}{(t+c)^{p}},
$$

where $t$ is the elapsed time after a mainshock, $K$ is the degree of the aftershock activity, $c$ is a parameter characterizing the relaxation time of the activity, and $p$ is the power-law exponent. In particular, it has been shown that the parameter $p$ clearly depends on the magnitude of the mainshock [40].

Third, we assume that background earthquakes occur independently of mainshocks and aftershocks. This assumption differs from the epidemic-type aftershock sequence model [9], where every earthquake is triggered by mainshocks or aftershocks, which are usually triggered by a mainshock. Furthermore, we assume that the rate of the background earthquakes depends on the magnitudes of the mainshocks; that is, when the magnitude of the mainshock is large, the rate of the background earthquakes is also large. It is difficult to determine whether an earthquake is an aftershock or a background 


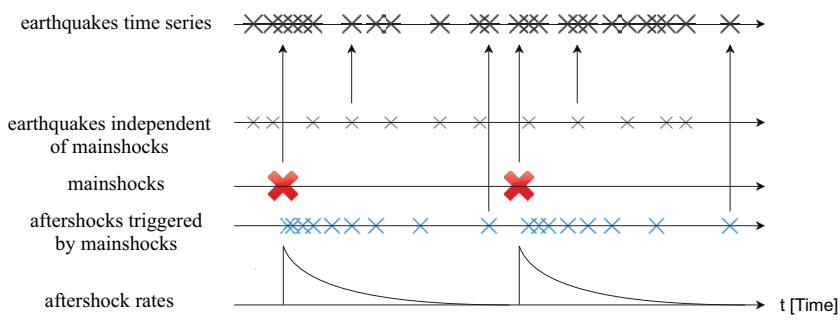

FIG. 2. Schematic view of earthquake model. Earthquake time series are obtained by superposing mainshocks, aftershocks, and background earthquakes. We assume that aftershock rate $\lambda_{a}(t)$ is the same for each mainshock.

earthquake. As we consider earthquakes over a vast region, we assume that almost all earthquakes are independent of mainshocks, and thus are background earthquakes. Under this assumption, we can determine some of the model parameters. Figure 2 shows a schematic view of our model.

A Poisson process with rate $\lambda$ can be generated by creating interevent times following the exponential distribution with mean $1 / \lambda$ in numerical simulations. In particular, the mainshocks and background earthquakes are generated by Poisson processes with rates $\lambda_{s}$ and $\lambda_{m}$, respectively. As aftershocks are described by Omori's law, we generate them by using a nonstationary Poisson process (see Appendix A for the details). Unlike a non-Markov model of aftershocks [41], it is a Markov model with the exception that it is nonstationary.

\section{DETRENDED FLUCTUATION ANALYSIS}

The DFA was invented to analyze data that have local trends and nonstationary features [28]. This method has been used to unravel long-range correlations in stationary as well as nonstationary time series such as heartbeat rates, weather variations, recurrence times of earthquakes, and conformation fluctuations of proteins [29,42-46].

The basic idea is to quantify fluctuations around local trends as

$$
F^{2}(n) \equiv \frac{1}{m n} \sum_{j=0}^{m-1} \sum_{i=j n+1}^{(j+1) n}\left(y_{i}-\tilde{y}_{i}^{j}\right)^{2},
$$

where $y_{i}$ is a time series that is considered, and $\tilde{y}_{i}^{j}$ represents a local trend in the time interval $[j n+1,(j+1) n]$. Thus, $n$ is the length of these time intervals. As the local trend $\tilde{y}_{i}^{j}$, we employ a linear function obtained by the least-squares fit to data $y_{i}$ in the interval $[j n+1,(j+1) n]$, i.e., we study the first-order DFA [31-33]. If higher-order DFAs are utilized, results in this paper will be modified.

Even when there are local trends in data, the function $F(n)$ characterizes a long-term correlation in the data. In particular, $F(n)$ increases as $F(n) \propto n^{1 / 2}$ when there is no correlation in increments $\Delta y_{i}$ of $y_{i}$, i.e., $\Delta y_{i} \equiv y_{i}-y_{i-1}$. However, it increases as $F(n) \propto n^{\alpha}$ with $\alpha \neq 1 / 2$ when the increment has a strong correlation, implying a power-law decay of the correlation function. In particular, $\alpha<1 / 2$ implies that there is an anticorrelation in increments $\Delta y_{i}$ and $\alpha>1 / 2$ implies a positive correlation of increments $\Delta y_{i}$

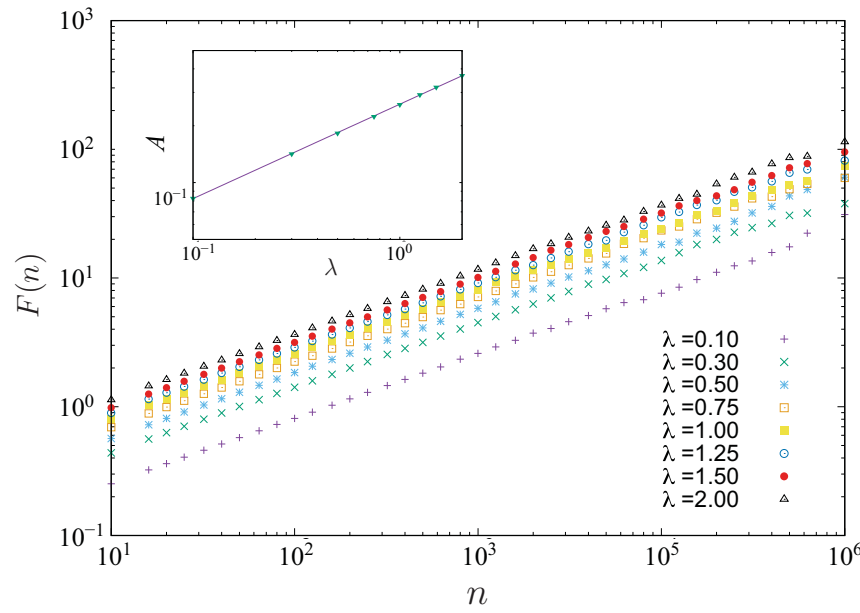

FIG. 3. Detrended fluctuation analysis of $N(t)$ in Poisson processes for different $\lambda$, where the total length of the time series is fixed at $10^{7}$. Inset: Constant $A$ as a function of $\lambda$. The solid line represents $A=\sqrt{\lambda} / \sqrt{15}$.

Here, we apply the DFA to time series $y_{i}$ generated by a point process. More precisely, $y_{i}$ is a monotonically increasing sequence defined by $y_{i}=N(i)$, where $N(i)$ is the cumulative number of earthquakes up to time $i$. The variable $i$ is an integer in the original DFA, whereas $i$ in $N(i)$ represents the continuous time; thus, it is a real number. Hence, in what follows, we use $t$ as the argument and use the notation $N(t)$. Note, however, that the definition of the function $F(n)$ in Eq. (2) remains unchanged even for point processes because we only use discrete data points of $N(t)$. In a previous study [47], the DFA for a sequence generated by a point process such as $N(t)$ was studied, where interoccurrence times are IID random variables. Such a process is called a renewal process. However, the interoccurrence times may not be IID and the time series are nonstationary of the first type in earthquakes. Here, we investigate the DFA for point processes for such nonstationary time series.

\section{DETRENDED FLUCTUATIONS ANALYSIS ON SYNTHESIZED DATA}

In this section, the DFA is applied to three types of data synthesized by the earthquake model: (1) background earthquakes (Poisson processes), (2) one mainshock and its aftershocks without background earthquakes, and (3) Poissonian mainshocks with aftershocks and background earthquakes. Numerical simulations are carried out for these models and compared with theoretical predictions for small and large $n$. Derivations of these predictions are presented in Appendixes $\mathrm{C}$ and $\mathrm{D}$.

\section{A. Background earthquakes (Poisson process)}

First, we apply the DFA to Poisson processes, for which interoccurrence times follow an exponential distribution with rate $\lambda$. Figure 3 shows that $F(n)$ increases as $F(n)=A n^{1 / 2}$ for any $n>0$ and a constant $A$ depends on rate $\lambda$ of the Poisson 
(a)

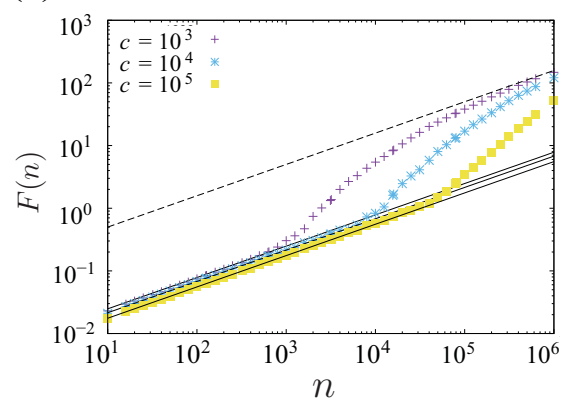

(b)

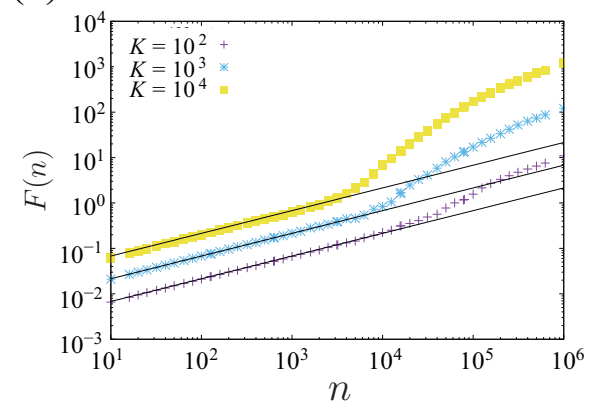

(c)

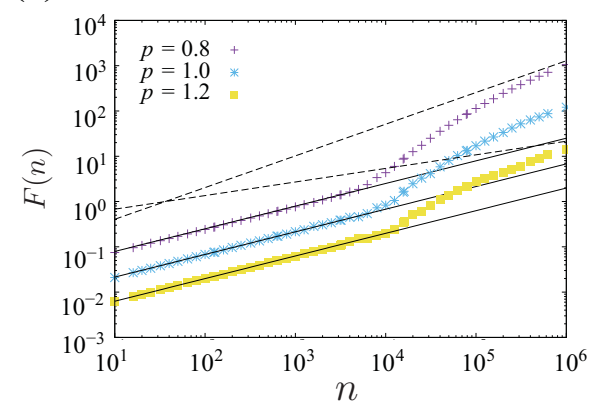

FIG. 4. Detrended fluctuation analysis (DFA) of $N(t)$ for earthquake model, where the length of time series is fixed as $10^{7}$. (a) DFAs for different values of $c$. The other parameters are fixed at $p=1$ and $K=10^{3}$. All the solid lines represent $F(n)=B n^{1 / 2}$ using Eq. (4). The dashed line represents Eq. (6). (b) DFAs for different $K$ values. The other parameters are fixed at $p=1$ and $c=10^{4}$. (c) DFAs for different values of $p$. The other parameters are fixed at $c=10^{4}$ and $K=10^{3}$. All the solid lines represent $F(n)=B n^{1 / 2}$ using Eq. (4). The dashed lines represent Eq. (7).

process. A theory of the DFA for a Poisson process implies

$$
A \cong \sqrt{\frac{\lambda}{15}}
$$

(a proof is given in Appendix C and see also Ref. [31]), which is confirmed using numerical simulations (inset of Fig. 3). As a Poisson process is a memoryless process, the scaling of $F(n) \propto n^{1 / 2}$ is quite reasonable. However, we numerically find that scaling $n^{1 / 2}$ is no longer valid for renewal processes where the PDF of interoccurrence times follows a power-law distribution with a divergent mean. Therefore, scaling $n^{1 / 2}$ represents the signature of a stationary Poisson process. In a biased continuous-time random walk, the variance of the displacement, which is a quantity similar to the DFA, shows an anomalous scaling [48,49].

\section{B. One mainshock and its aftershocks without background earthquakes}

Second, synthesized data $N(t)$ are generated using the earthquake model. To obtain a deeper understanding of the features of the DFA, we consider a simple situation where a mainshock occurs only once at $t=0$, and there are no background earthquakes; i.e., the time series comprises one mainshock and its aftershocks.

As shown in Fig. 4, all the results of the DFA show a crossover from $n^{1 / 2}$ to $n^{\alpha}$ scaling. For small- $n$ behavior, $F(n)$ shows $F(n)=B n^{1 / 2}$. By an adiabatic approximation, we approximately obtain $B$ :

$$
B \cong \frac{\sqrt{\overline{\lambda_{a}}}}{\sqrt{15}}
$$

where

$$
\overline{\lambda_{a}}=\frac{1}{T} \int_{0}^{T} \lambda_{a}(t) d t
$$

and $T$ is the total length of the time series. For large- $n$ behavior, $F(n)$ also shows

$$
F(n) \sim \frac{K}{2 \sqrt{T}} n^{1 / 2}
$$

when $p=1$ (see Appendix D). Equation (6) is a special case of a general result for $p<3 / 2$,

$$
F(n) \sim \frac{K p}{\sqrt{T(3-2 p)}(3-p)(2-p)} n^{3 / 2-p},
$$

which is valid for $n \rightarrow \infty$ (see Appendix D). Thus, the powerlaw exponent in the DFA is determined by $p$. In other words, the parameter $p$ can be obtained from the asymptotic behavior of the DFA for $N(t)$. This is one of the most important analytical results of our study.

Figure 4 summarizes the results of the DFAs of $N(t)$ for different parameters. Figure 4(a) shows that crossover time $n_{c}$ in $F(n)$ increases with increasing parameter $c$ and short- $n$ behaviors are almost the same. For large- $n$ behavior, $F(n)$ converges to an $n^{1 / 2}$ scaling, which does not depend on $c$. As shown in Figs. 4(b) and 4(c), crossover time $n_{c}$ also depends on $K$ and $p$, but the dependencies are relatively weak compared with the $c$ dependency. Importantly, crossover time $n_{c}$ is thus almost proportional to $c$. Therefore, the parameter $c$ can be estimated from the crossover time $n_{c}$. This is significantly important when time series are a superposition of nonstationary and stationary signals because information of the nonstationary part can be obtained without distinguishing the stationary and nonstationary time series. In Fig. 4, it is clearly shown that the asymptotic behaviors of $F(n)$ exhibit different power-law scaling with exponent $3 / 2-p$. Therefore, the parameter $p$ can be obtained from the asymptotic behavior of the DFA for $N(t)$ if background earthquakes are removed from the time series.

\section{Poissonian mainshocks with aftershocks and background earthquakes}

For synthesized data $N(t)$ generated by the earthquake model with several mainshocks, we find a crossover phenomenon such that $F(n)$ exhibits an $n^{1 / 2}$ to $n^{\alpha}$ scaling. The superposition of two Poisson processes with rates $\lambda_{1}$ and $\lambda_{2}$ is equivalent to a Poisson process with rate $\lambda_{1}+\lambda_{2}$. Therefore, the DFA for the superposition of the two Poisson processes with rates $\lambda_{1}$ and $\lambda_{2}$ becomes $F(n)=\sqrt{\left(\lambda_{1}+\lambda_{2}\right) n} / \sqrt{15}$. The synthesized time series are composed of background earthquakes and aftershocks triggered by a mainshock. Since 
(a)

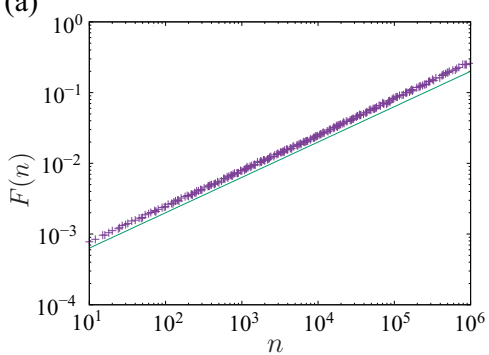

(c)

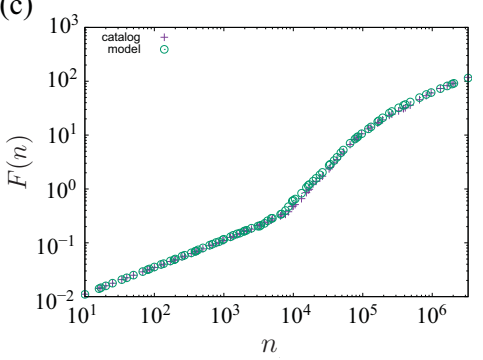

(b)

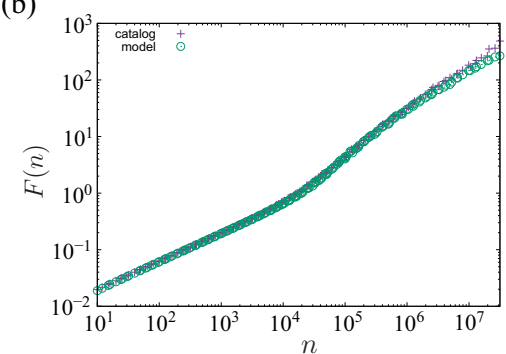

(d)

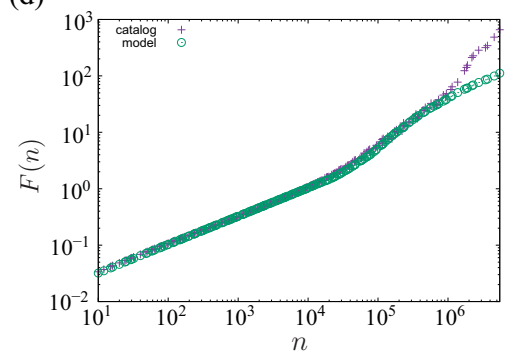

(e)

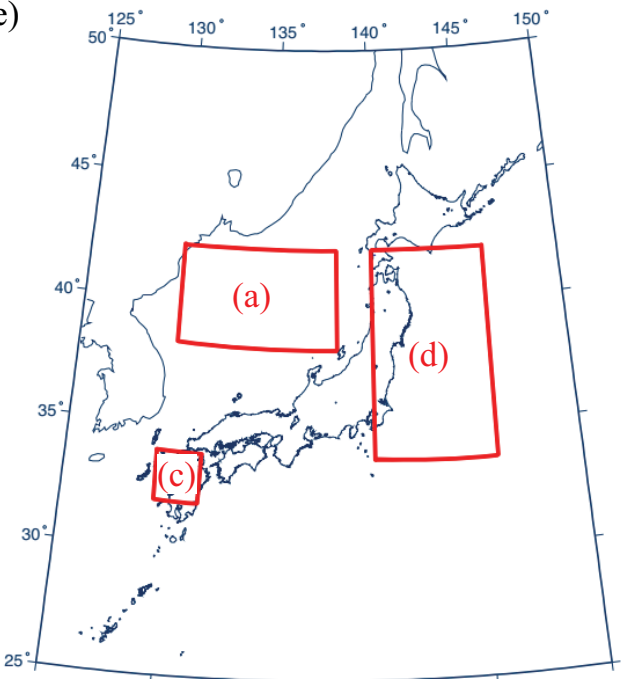

FIG. 5. Detrended fluctuation analysis (DFA) of $N(t)$ for earthquake data, where the sampling interval is $1 \mathrm{~s}$. Crosses are the results for the catalog data (real earthquakes), and circles are those for data synthesized with the earthquake models. (a) DFA for time series $N(t)$ with no mainshock at some place. $N(t)$ is obtained with earthquakes occurred inside the area enclosed within $38^{\circ}-42^{\circ} \mathrm{N}$ latitude and $130^{\circ}-138^{\circ}$ E longitude [see (e)]. The period of time series $N(t)$ is restricted from 1 January 2009 to 31 December 2010. (b) DFA for time series $N(t)$ with several mainshocks. $N(t)$ is obtained with earthquakes occurred inside the area enclosed within $25^{\circ}-50^{\circ} \mathrm{N}$ latitude and $125^{\circ}-150^{\circ} \mathrm{E}$ longitude. The parameters of the earthquake model are $\lambda_{s}=5.0 \times 10^{-4}, c=10^{4}, K=10^{3}$, and $p=1.15$. The period of time series $N(t)$ is restricted from 1 January 2001 to 31 December 2010. (c) DFA for Kumamoto earthquakes. $N(t)$ is obtained with earthquakes occurred inside the area enclosed within $32^{\circ}-34^{\circ} \mathrm{N}$ latitude and $129.5^{\circ}-131.5^{\circ} \mathrm{E}$ longitude [see (e)]. The period of time series $N(t)$ is restricted from 16 April 2016 to 30 April 2017. The parameters of the earthquake model are $\lambda_{s}=1.5 \times 10^{-5}, c=10^{4}, K=5.0 \times 10^{3}$, and $p=1.15$. (d) DFA for Tohoku earthquakes. $N(t)$ is obtained with earthquakes occurred inside the area enclosed within $34^{\circ}-42^{\circ} \mathrm{N}$ latitude and $140^{\circ}-146^{\circ} \mathrm{E}$ longitude [see (e)]. The period of time series $N(t)$ is restricted from 11 March 2011 to 6 December 2012. The parameters of the earthquake model are $\lambda_{s}=1.5 \times 10^{-3}, c=3.0 \times 10^{4}, K=10^{4}$, and $p=1.2$. (e) Three regions used for the analysis of the subplots (a), (c), and (d). The entire region is used for the analysis of subplot (b).

background earthquakes and mainshocks are described by Poisson processes with rates $\lambda_{b}$ and $\lambda_{m}$, the above estimation can be utilized. For small- $n$ behavior, $F(n)$ shows that $F(n) \simeq C n^{1 / 2}$ and $C$ can be approximately obtained as

$$
C \cong \frac{\sqrt{\lambda_{b}+\lambda_{m} \times N_{a}}}{\sqrt{15}},
$$

where

$$
N_{a}=\int_{0}^{1 / \lambda_{m}} \lambda_{a}(t) d t .
$$

The small- $n$ behavior of the DFA for $N(t)$ is determined by $\lambda_{b}, \lambda_{m}$, and $N_{a}$.

\section{DETRENDED FLUCTUATION ANALYSIS ON EARTHQUAKE DATA CATALOG}

Here, we apply the DFA to the cumulative number $N(t)$ of real earthquakes included in the JMA catalog; this catalog contains data of earthquakes with magnitude $M \geqslant 2$ and the ones that occurred in the area of $25^{\circ}-50^{\circ} \mathrm{N}$ latitude and $125^{\circ}-$ $150^{\circ}$ E longitude. Figure 5 shows DFAs for $N(t)$ for several different periods and areas in Japan. We find a crossover phenomenon in $F(n)$; i.e., $F(n)$ increases as $F(n) \propto n^{1 / 2}$ for small $n$ and $F(n)$ shows another scaling for large $n$ if the data are affected by at least one mainshock. More precisely,
$F(n)$ increases as $F(n) \propto n^{\alpha}$ for $n>n_{c}$, where $\alpha \cong 1.0$ and $n_{c} \cong 1.6 \times 10^{4} \mathrm{~s}(=4.5 \mathrm{~h})$. This crossover is observed for all earthquake time series if they contain mainshocks, i.e., earthquakes with magnitudes greater than 7. Moreover, small$n$ behaviors of $F(n)$ are almost equivalent for all data, while the crossover times $n_{c}$ are slightly different. Furthermore, we observe that synthesized data $N(t)$ generated by the earthquake model, where parameters are set to be $\lambda_{s}^{-1}=2.0 \times 10^{3}$ $\mathrm{s}, c=10^{4} \mathrm{~s}, K=10^{3}$, and $p=1.15$, show similar crossover phenomena in the DFA [see Fig. 5(b)]. In these synthesized data, instead of generating mainshocks according to Poisson statistics, mainshocks are assumed to occur at the same times $t_{i}(i=1,2, \ldots)$ at which real earthquakes with magnitudes greater than 7 occurred.

Furthermore, we applied the DFA to $N(t)$ for specific earthquakes such as the Tohoku and Kumamoto earthquakes. In the Tohoku earthquakes, a mainshock occurred on 11 March 2011 with a magnitude of $M=9.0$ and we analyzed earthquakes after the mainshock whose area overlaps the area around the epicenter [see Fig. 5(e)]. In the Kumamoto earthquakes, a mainshock occurred on 16 April 2016 with a magnitude of $M=7.3$, and we analyze earthquakes after the mainshock whose area overlaps the area around the epicenter [see Fig. 5(e)]. In the DFAs for these real earthquakes [Figs. 5(c) and 5(d)], we find crossover phenomena similar to that found for the synthesized data. Moreover, we successfully generate 
a time series $N(t)$ with our earthquake model that reproduces the DFAs of the two earthquake time series, i.e., the Tohoku and Kumamoto earthquakes [circles in Figs. 5(c) and 5(d)]. In the DFA of the Tohoku earthquakes at large $n$, however, there is a slight difference between the results of the catalog data and the earthquake model. While we assume that a mainshock occurs only at $t=0$, there are a few mainshocks (earthquakes with magnitudes greater than 7) occurring after $t=0$ in the catalog data. We believe that such large aftershocks significantly affect subsequent aftershocks. Therefore, our model cannot completely reproduce the DFA of Tohoku earthquakes.

\section{CONCLUSION}

We found that crossover phenomena in the DFA of $N(t)$, i.e., the number of earthquakes up to time $t$, are universally observed in earthquake data. Extensive numerical simulations of the earthquake model show that the crossover phenomena originate from the nonstationarity of the aftershock sequences. In particular, crossover time $n_{c}$ in the DFA characterizes parameter $c$, which represents the relaxation time of aftershocks in Omori's law. Although we do not determine if an earthquake is an aftershock or not, we can successfully obtain information regarding the aftershocks. In addition, our analysis is not restricted to the earthquake phenomenon. Therefore, it is significantly important when the time series is a superposition of the two types of time series that cannot be distinguished and when one of the two types is nonstationary and the other is stationary. Moreover, we present theories of the DFA for stationary [Eq. (3)] and nonstationary point processes [Eq. (7)], which are necessary for performing a thorough analysis. However, we note that these theoretical results will be changed if we use the higher-order DFA. Future work is therefore needed to determine the differences between the first and the higher-order DFA for point processes.

\section{ACKNOWLEDGMENTS}

T.A. was supported by JSPS Grant-in-Aid for Scientific Research (Grant No. C JP18K03468).

\section{APPENDIX A: NUMERICAL SIMULATIONS OF NONSTATIONARY POISSON PROCESSES}

In numerical simulations of the earthquake model in Sec. II, aftershock sequences, which follow a nonstationary Poisson process, must be generated. Aftershocks triggered by a mainshock are generated as follows: Here, we assume that a mainshock occurs at $t=-t_{0}\left(t_{0}>0\right)$. First, time $t_{0}$ can be determined as follows: As mainshocks are described by a Poisson process with rate $\lambda_{m}, t_{0}$ can be obtained by generating a random variable following the exponential distribution with rate $\lambda_{m}$. This is because the random variable $t_{0}$, which is called the backward recurrence time in renewal theory [35], follows the same exponential distribution as the interoccurrence-time distribution of mainshocks as a result of the memoryless property of Poisson processes [35]. In computer programs, $t_{0}$ is obtained by $t_{0}=-\lambda_{m}^{-1} \ln X$, where $X$ is a random variable uniformly distributed on $[0,1]$.
The probability that an aftershock triggered by the mainshock at $t=-t_{0}$ occurs at $t=0$ is given by $\lambda_{a}\left(t_{0}\right) \Delta t$, where $\Delta t$ is a time step. In the JMA earthquake catalog, time step $\Delta t$ is $\Delta t=1 \mathrm{~s}$. In computer programs, a random variable $X$ is uniformly distributed on $[0,1]$. Then, we generate an aftershock at $t=0$ if $X<\lambda_{a}\left(t_{0}\right) \Delta t$, and no aftershock occurs at $t=0$ otherwise. This procedure is repeated for $t=\Delta t, 2 \Delta t, \ldots$ with rates $\lambda_{a}\left(t_{0}+\Delta t\right), \lambda_{a}\left(t_{0}+2 \Delta t\right), \ldots$ In particular, the probability that an aftershock triggered by the mainshock occurs at $t=n \Delta t$ is given by $\lambda_{a}\left(t_{0}+n \Delta t\right) \Delta t$.

For simplicity, we generate aftershocks triggered by the mainshock at $t=-t_{0}$ until the next mainshock occurs at $t=t_{1}$. This is because the rate of aftershocks triggered by the next mainshock $\lambda_{a}\left(t-t_{1}\right)$ is much greater than that triggered by the previous mainshock $\lambda_{a}\left(t+t_{0}\right)$ where $t>t_{1}$. Similar procedures were employed for the subsequent mainshocks at $t=t_{2}, t_{3}, \ldots$ and their aftershocks.

\section{APPENDIX B: DIMENSIONLESS FORM OF OCCURRENCE RATE}

Here, we transform the time-dependent occurrence rate $\lambda(t)$ [Eq. (1)] in a dimensionless form. By the following transformation,

$$
\begin{aligned}
t & \rightarrow t / c \equiv \tilde{t}, \quad \lambda_{a}(t) \rightarrow c \lambda_{a}(t) \equiv \tilde{\lambda}_{a}(\tilde{t}), \\
K & \rightarrow c^{1-p} K \equiv \tilde{K},
\end{aligned}
$$

we have a nondimensional occurrence rate

$$
\tilde{\lambda}_{a}(\tilde{t})=\frac{\tilde{K}}{(\tilde{t}+1)^{p}} .
$$

In addition, if the measurement time $T$ is transformed as $T \rightarrow$ $T / c \equiv \tilde{T}$, the remaining parameters are $\tilde{K}, \tilde{T}$, and $p$.

\section{APPENDIX C: THEORY OF DFA FOR POINT PROCESS}

In this study, our objective is the extraction of nonstationary information from point processes using DFA. Here, we provide a theoretical argument regarding the DFA for stationary point processes with constant rate $\lambda$.

\section{Interoccurrence-time distribution}

We assume that interoccurrence-time distribution of successive renewals follows a distribution $\psi(\tau)$ with finite mean and variance $\langle\tau\rangle$ and $\left\langle\tau^{2}\right\rangle-\langle\tau\rangle^{2}$. For this interoccurrencetime distribution, the mean and variance of $N(i)$ are given by $[35,50]$

$$
\begin{gathered}
\langle N(i)\rangle \sim \frac{i}{\langle\tau\rangle}, \\
\left\langle N^{2}(i)\right\rangle-\langle N(i)\rangle^{2} \sim \frac{\left\langle\tau^{2}\right\rangle-\langle\tau\rangle^{2}}{\langle\tau\rangle^{3}} i .
\end{gathered}
$$

In particular, the mean interval $\langle\tau\rangle$ is related with the rate $\lambda$ as $\langle\tau\rangle=1 / \lambda$; thus, Eq. $(\mathrm{C} 1)$ is rewritten as $\langle N(i)\rangle \sim \lambda i$. For Eq. (C2), we also use a notation $\left\langle N^{2}(i)\right\rangle-\langle N(i)\rangle^{2}=\sigma^{2} i$, where $\sigma^{2}$ is defined by $\sigma^{2}=\left(\left\langle\tau^{2}\right\rangle-\langle\tau\rangle^{2}\right) /\langle\tau\rangle^{3}$. 
If $\psi(\tau)$ is given by the exponential distribution

$$
\psi(\tau)=\frac{1}{\langle\tau\rangle} e^{-\tau /\langle\tau\rangle},
$$

the point process is referred to as the Poisson process. For the Poisson process Eqs. (C1) and (C2) are given by

$$
\begin{gathered}
\langle N(i)\rangle=\frac{i}{\langle\tau\rangle}, \\
\left\langle N^{2}(i)\right\rangle-\langle N(i)\rangle^{2}=\frac{i}{\langle\tau\rangle} .
\end{gathered}
$$

Note that equalities hold for the Poisson process. From the definition of $\sigma^{2}$, it follows that $\sigma^{2}=\lambda$ holds for the Poisson process.

\section{Theory of DFA for point process}

Based on the stationarity of the point process, Eq. (2) can be represented as

$$
F^{2}(n)=\left\langle\frac{1}{n} \sum_{i=1}^{n}\left(y_{i}-\tilde{y}_{i}^{0}\right)^{2}\right\rangle,
$$

where $y_{i}=N(i)$ is obtained using the point process described above. We rewrite the DFA as

$$
\begin{aligned}
F^{2}(n) & =\frac{1}{n} \sum_{i=1}^{n}\left\langle\left[y_{i}-\lambda i-\left(\tilde{y}_{i}^{0}-\lambda i\right)\right]^{2}\right\rangle \\
& =\frac{1}{n} \sum_{i=1}^{n}\left[\tilde{y}_{i}-(a i+b)\right]^{2},
\end{aligned}
$$

where $\tilde{y}$ is defined as $\tilde{y}_{i}=y_{i}-\lambda i$. Moreover, $a$ and $b$ are the coefficients of the linear fitting of $\tilde{y}_{i}$ by the least-squares method, i.e., $\tilde{y}_{i}^{0}-\lambda i=a i+b$. By expanding the summand,

we obtain

$$
\begin{aligned}
F^{2}(n)= & \frac{1}{n} \sum_{i=1}^{n}\left(\left\langle\tilde{y}_{i}^{2}\right\rangle-2 i\left\langle a \tilde{y}_{i}\right\rangle-2\left\langle b \tilde{y}_{i}\right\rangle\right. \\
& \left.+i^{2}\left\langle a^{2}\right\rangle+2 i\langle a b\rangle+\left\langle b^{2}\right\rangle\right) .
\end{aligned}
$$

The parameters $a$ and $b$ are given by

$$
a=\frac{S_{x y}}{S_{x}^{2}}-\lambda, \quad b=\frac{1}{n}-\frac{n+1}{2} \frac{S_{x y}}{S_{x}^{2}},
$$

where $S_{x y}$ and $S_{x}^{2}$ are a covariance and a variance given by

$$
\begin{gathered}
S_{x y}=\frac{1}{n} \sum_{j=1}^{n} j y_{j}-\frac{1}{n^{2}} \sum_{j=1}^{n} j \sum_{k=1}^{n} y_{k}, \\
S_{x}^{2}=\frac{1}{n} \sum_{j=1}^{n} j^{2}-\frac{1}{n^{2}}\left(\sum_{j=1}^{n} j\right)^{2} .
\end{gathered}
$$

It follows that $a$ and $b$ are written as

$$
\begin{gathered}
a=\frac{1}{n S_{x}^{2}} \sum_{j=1}^{n}\left(j-\frac{n+1}{2}\right) \tilde{y}_{j}, \\
b=\frac{1}{n} \sum_{j=1}^{n} \tilde{y}_{j}-\frac{n+1}{2} a .
\end{gathered}
$$

In Poisson processes, we have $\left\langle N(i) N\left(i+i^{\prime}\right)\right\rangle=\left\langle N^{2}(i)\right\rangle+$ $\left\langle N(i)\left[N\left(i^{\prime}\right)-N(i)\right]\right\rangle=\left\langle N^{2}(i)\right\rangle+\langle N(i)\rangle\left\langle N\left(i^{\prime}\right)-N(i)\right\rangle$, because a Poisson process is a memoryless process, i.e., $N(i)$ and $N\left(i^{\prime}\right)-N(i)$ are independent. It follows that $\left\langle\tilde{y}_{i}\right\rangle=0$, $\left\langle\tilde{y}_{i}^{2}\right\rangle=\sigma^{2} i$, and $\left\langle\tilde{y}_{i} \tilde{y}_{j}\right\rangle=\sigma^{2} \min (i, j)$. For general point processes, $N(i)$ and $N\left(i^{\prime}\right)-N(i)$ are not independent. It becomes

$$
\left\langle\tilde{y}_{i} \tilde{y}_{j}\right\rangle \approx \frac{i+j}{2} \sigma^{2}-\frac{|i-j|}{2} \lambda
$$

By using Eqs. (C13) and (C15), we obtain

$$
\sum_{i=1}^{n}\left(i-\frac{n+1}{2}\right)\left\langle\tilde{y}_{i} a\right\rangle \approx \frac{-\lambda}{n S_{x}^{2}} \sum_{i, j=1}^{n}\left(i-\frac{n+1}{2}\right)\left(j-\frac{n+1}{2}\right) \frac{|i-j|}{2}
$$

and

$$
\left\langle a^{2}\right\rangle=\frac{-\lambda}{\left(n S_{x}^{2}\right)^{2}} \sum_{i, j=1}^{n}\left(i-\frac{n+1}{2}\right)\left(j-\frac{n+1}{2}\right) \frac{|i-j|}{2},
$$

where the term $(i+j) \sigma^{2} / 2$ in Eq. (C15) vanishes considering the summation over $i$ and $j$, because $\sum_{i=1}^{n}[i-(n+1) / 2]=0$. By Eqs. (C15)-(C17), it follows that the DFA in Eq. (C9) is rewritten as

$$
\begin{aligned}
F^{2}(n)= & \frac{1}{n} \sum_{i=1}^{n} \sigma^{2} i-\frac{2}{n}\left[\sum_{i=1}^{n}\left(i-\frac{n+1}{2}\right)\left\langle a \tilde{y}_{i}\right\rangle+\frac{1}{n} \sum_{i, j=1}^{n}\left\langle\tilde{y}_{i} \tilde{y}_{j}\right\rangle\right] \\
& +\frac{1}{n} \sum_{i=1}^{n}\left[\left(i-\frac{n+1}{2}\right)^{2}\left\langle a^{2}\right\rangle+\frac{2}{n} \sum_{j=1}^{n}\left(i-\frac{n+1}{2}\right)\left\langle a \tilde{y}_{j}\right\rangle+\frac{1}{n^{2}} \sum_{j, k=1}^{n}\left\langle\tilde{y}_{j} \tilde{y}_{k}\right\rangle\right] \\
\simeq & \frac{\sigma^{2} n}{2}+\frac{\lambda}{n^{2} S_{x}^{2}} \sum_{j, k=1}^{n}\left(j-\frac{n+1}{2}\right)\left(k-\frac{n+1}{2}\right) \frac{|k-j|}{2}-\frac{\sigma^{2} n}{2}+\frac{n}{6} \lambda \\
\simeq & \frac{\lambda n}{15}
\end{aligned}
$$


where we used an approximation, $\sum_{j=1}^{n} j^{k} \simeq n^{k+1} /(k+1)$ for $n \rightarrow \infty$. Equation (C18) is valid for any point process that has a finite mean and variance. Thus, Eq. (C18) holds for the Poisson processes, and thus we obtain Eq. (3).

\section{APPENDIX D: ASYMPTOTIC BEHAVIOR OF THE DFA FOR AFTERSHOCK SEQUENCES}

Here, we evaluate the asymptotic behavior of the DFA for aftershock sequences in the large- $n$ limit. The mean number of aftershocks is given by $\langle N(t)\rangle=\int_{0}^{t} \lambda_{a}\left(t^{\prime}\right) d t^{\prime}$. Because the derivative of $\langle N(t)\rangle$ tends to zero for $t \rightarrow \infty$, deviations from a linear fitting become zero for the large- $t$ limit. In other words, the deviation in the first time window in $F(n)$ is significant in the large- $n$ limit. Therefore, in the large- $n$ limit, $F(n)$ can be approximately obtained from the first time window:

$$
F(n)^{2} \cong \chi_{0}(n)^{2} \equiv \frac{1}{n} \sum_{i=1}^{n}\left(y_{i}-\tilde{y}_{i}^{0}\right)^{2}
$$

In the following, we replace $y_{i}$ with $\langle N(t)\rangle$ to calculate $\chi_{0}(n)^{2}$. Using the least mean square method, we have a linear function, i.e., $\tilde{y}_{i}^{0} \equiv a i+b$.

For $p=1,\langle N(t)\rangle$ is given by

$$
\langle N(t)\rangle=K \log \left(\frac{t}{c}+1\right) .
$$

Using $\partial \chi_{0}(n)^{2} / \partial a=\partial \chi_{0}(n)^{2} / \partial b=0$, we obtain

$$
a=\frac{3 K}{n}, \quad b=K\left[\log \left(\frac{n}{c}+1\right)-\frac{5}{2}\right] .
$$

We approximate the sum in Eq. (D1) by the integral

$$
\chi_{0}(n)^{2} \cong \frac{1}{T} \int_{0}^{n}\left[K \log \left(\frac{t+c}{n+c}\right)-\frac{3 K}{n} t-\frac{5 K}{2}\right]^{2} .
$$

For $n \gg c$, we have

$$
F(n) \cong \frac{K}{2} \sqrt{\frac{n}{T}}
$$

For $p \neq 1,\langle N(t)\rangle$ is given by

$$
\langle N(t)\rangle=\frac{c^{1-p} K}{1-p}\left[\left(\frac{t}{c}+1\right)^{1-p}-1\right] .
$$

In the long-t limit, $\langle N(t)\rangle$ becomes

$$
\langle N(t)\rangle \sim \frac{K}{1-p} t^{1-p}
$$

and

$$
\langle N(t)\rangle-\frac{c^{1-p} K}{p-1} \sim-\frac{K}{p-1} t^{1-p}
$$

for $p<1$ and $p>1$, respectively. In the same calculation as the above, we have

$$
F(n) \cong \frac{K p}{\sqrt{T(3-2 p)}(3-p)(2-p)} n^{\frac{3}{2}-p}
$$

for $p<3 / 2(p \neq 1)$.
[1] H. Scher and E. W. Montroll, Anomalous transit-time dispersion in amorphous solids, Phys. Rev. B 12, 2455 (1975).

[2] J.-P. Bouchaud and A. Georges, Anomalous diffusion in disordered media: Statistical mechanisms, models and physical applications, Phys. Rep. 195, 127 (1990).

[3] J.-P. Bouchaud, Weak ergodicity breaking and aging in disordered systems, J. Phys. I (France) 2, 1705 (1992).

[4] C. Monthus and J.-P. Bouchaud, Models of traps and glass phenomenology, J. Phys. A 29, 3847 (1996).

[5] X. Brokmann, J. P. Hermier, G. Messin, P. Desbiolles, J. P. Bouchaud, and M. Dahan, Statistical Aging and Nonergodicity in the Fluorescence of Single Nanocrystals, Phys. Rev. Lett. 90, 120601 (2003).

[6] R. Metzler, J.-H. Jeon, A. G. Cherstvy, and E. Barkai, Anomalous diffusion models and their properties: Non-stationarity, non-ergodicity, and ageing at the centenary of single particle tracking, Phys. Chem. Chem. Phys. 16, 24128 (2014).

[7] F. Omori, On the aftershocks of earthquakes, J. Coll. Sci. Imp. Univ. Tokyo 7, 111 (1894).

[8] T. Utsu, Aftershocks and earthquake statistics II: Further investigation of aftershocks and other earthquake sequence based on a new classification of earthquake sequences, J. Fac. Sci. Hokkaido Univ. Ser. VII 3, 197 (1970).

[9] Y. Ogata, Statistical models for earthquake occurrence and residual analysis for point processes, J. Am. Stat. Assoc. 83, 9 (1988).
[10] T. Utsu, A review of seismicity (in Japanese), in Mathematical Seismology, edited by M. Saito, Vol. 2 (Institute of Statistical Mathematics, Tokyo, 1992).

[11] A. Weigel, B. Simon, M. Tamkun, and D. Krapf, Ergodic and nonergodic processes coexist in the plasma membrane as observed by single-molecule tracking, Proc. Natl. Acad. Sci. U.S.A. 108, 6438 (2011).

[12] E. Yamamoto, T. Akimoto, M. Yasui, and K. Yasuoka, Origin of subdiffusion of water molecules on cell membrane surfaces, Sci. Rep. 4, 4720 (2014).

[13] C. Manzo, J. A. Torreno-Pina, P. Massignan, G. J. Lapeyre, Jr., M. Lewenstein, and M. F. Garcia Parajo, Weak Ergodicity Breaking of Receptor Motion in Living Cells Stemming from Random Diffusivity, Phys. Rev. X 5, 011021 (2015).

[14] T. Akimoto, E. Barkai, and G. Radons, Infinite invariant density in a semi-Markov process with continuous state variables, Phys. Rev. E 101, 052112 (2020).

[15] B. Gutenberg and C. F. Richter, Frequency of earthquakes in California, Bull. Seismol. Soc. Am. 34, 185 (1944).

[16] K. Z. Nanjo, N. Hirata, K. Obara, and K. Kasahara, Decadescale decrease in $\mathrm{b}$ value prior to the M9-class 2011 Tohoku and 2004 Sumatra quakes, Geophys. Res. Lett. 39, L20304 (2012).

[17] Y. He, S. Burov, R. Metzler, and E. Barkai, Random Time-Scale Invariant Diffusion and Transport Coefficients, Phys. Rev. Lett. 101, 058101 (2008). 
[18] T. Miyaguchi and T. Akimoto, Intrinsic randomness of transport coefficient in subdiffusion with static disorder, Phys. Rev. E 83, 031926 (2011).

[19] T. Miyaguchi and T. Akimoto, Anomalous diffusion in a quenched-trap model on fractal lattices, Phys. Rev. E 91, 010102(R) (2015).

[20] T. Akimoto and E. Yamamoto, Distributional behavior of diffusion coefficients obtained by single trajectories in annealed transit time model, J. Stat. Mech. (2016) 123201.

[21] I. Y. Wong, M. L. Gardel, D. R. Reichman, E. R. Weeks, M. T. Valentine, A. R. Bausch, and D. A. Weitz, Anomalous Diffusion Probes Microstructure Dynamics of Entangled f-Actin Networks, Phys. Rev. Lett. 92, 178101 (2004).

[22] M. Kuno, D. P. Fromm, H. F. Hamann, A. Gallagher, and D. J. Nesbitt, Nonexponential "blinking" kinetics of single CdSe quantum dots: A universal power law behavior, J. Chem. Phys. 112, 3117 (2000).

[23] A. Corral, Long-Term Clustering, Scaling, and Universality in the Temporal Occurrence of Earthquakes, Phys. Rev. Lett. 92, 108501 (2004).

[24] S. Abe and N. Suzuki, Scale-free statistics of time interval between successive earthquakes, Physica A 350, 588 (2005).

[25] A. Saichev and D. Sornette, "Universal" Distribution of Interearthquake Times Explained, Phys. Rev. Lett. 97, 078501 (2006).

[26] T. Hasumi, T. Akimoto, and Y. Aizawa, The Weibull-log Weibull distribution for interoccurrence times of earthquakes, Physica A 388, 491 (2009).

[27] H. Tanaka and Y. Aizawa, Detailed analysis of the interoccurrence time statistics in seismic activity, J. Phys. Soc. Jpn. 86, 024004 (2017).

[28] C.-K. Peng, S. V. Buldyrev, S. Havlin, M. Simons, H. E. Stanley, and A. L. Goldberger, Mosaic organization of DNA nucleotides, Phys. Rev. E 49, 1685 (1994).

[29] S. Lennartz, V. Livina, A. Bunde, and S. Havlin, Long-term memory in earthquakes and the distribution of interoccurrence times, Europhys. Lett. 81, 69001 (2008).

[30] M. S. Taqqu, V. Teverovsky, and W. Willinger, Estimators for long-range dependence: An empirical study, Fractals 03, 785 (1995).

[31] M. Höll and H. Kantz, The relationship between the detrendend fluctuation analysis and the autocorrelation function of a signal, Euro. Phys. J. B 88, 327 (2015).

[32] K. Kiyono, Establishing a direct connection between detrended fluctuation analysis and fourier analysis, Phys. Rev. E 92, 042925 (2015).

[33] M. Höll, K. Kiyono, and H. Kantz, Theoretical foundation of detrending methods for fluctuation analysis such as detrended fluctuation analysis and detrending moving average, Phys. Rev. E 99, 033305 (2019).
[34] M. Magdziarz, A. Weron, K. Burnecki, and J. Klafter, Fractional Brownian Motion Versus the Continuous-Time Random Walk: A Simple Test for Subdiffusive Dynamics, Phys. Rev. Lett. 103, 180602 (2009).

[35] D. R. Cox, Renewal Theory (Methuen, London, 1962).

[36] J. Gardner and L. Knopoff, Is the sequence of earthquakes in Southern California, with aftershocks removed, Poissonian? Bull. Seismol. Soc. Am. 64, 1363 (1974).

[37] Y. Y. Kagan and D. D. Jackson, Long-term earthquake clustering, Geophys. J. Int. 104, 117 (1991).

[38] Japan Meteorological Agency Earthquake Catalog, http:// evrrss.eri.u-tokyo.ac.jp/tseis/jma1/index.html.

[39] X. Yang, S. Du, and J. Ma, Do Earthquakes Exhibit SelfOrganized Criticality? Phys. Rev. Lett. 92, 228501 (2004).

[40] G. Ouillon and D. Sornette, Magnitude-dependent Omori law: Theory and empirical study, J. Geophys. Res. 110, B04306 (2005).

[41] T. Akimoto and Y. Aizawa, Large fluctuations in the stationarynonstationary chaos transition, Prog. Theor. Phys. 114, 737 (2005).

[42] C.-K. Peng, J. Mietus, J. M. Hausdorff, S. Havlin, H. E. Stanley, and A. L. Goldberger, Long-Range Anticorrelations and NonGaussian Behavior of the Heartbeat, Phys. Rev. Lett. 70, 1343 (1993).

[43] E. Koscielny-Bunde, A. Bunde, S. Havlin, H. E. Roman, Y Goldreich, and H.-J. Schellnhuber, Indication of a Universal Persistence Law Governing Atmospheric Variability, Phys. Rev. Lett. 81, 729 (1998).

[44] S. Havlin, S. Buldyrev, A. Bunde, A. Goldberger, P. C. Ivanov, C.-K. Peng, and H. E. Stanley, Scaling in nature: From DNA through heartbeats to weather, Physica A 273, 46 (1999).

[45] T. Harada, T. Yokogawa, T. Miyaguchi, and H. Kori, Singular behavior of slow dynamics of single excitable cells, Biophys. J. 96, 255 (2009).

[46] E. Yamamoto, T. Akimoto, Y. Hirano, M. Yasui, and K. Yasuoka, 1/ $f$ fluctuations of amino acids regulate water transportation in aquaporin 1, Phys. Rev. E 89, 022718 (2014).

[47] P. Paradisi, R. Cesari, A. Donateo, D. Contini, and P. Allegrini, Scaling laws of diffusion and time intermittency generated by coherent structures in atmospheric turbulence, Nonlinear Proc. Geophys. 19, 113 (2012).

[48] T. Akimoto, A. G. Cherstvy, and R. Metzler, Ergodicity, rejuvenation, enhancement, and slow relaxation of diffusion in biased continuous-time random walks, Phys. Rev. E 98, 022105 (2018).

[49] R. Hou, A. G. Cherstvy, R. Metzler, and T. Akimoto, Biased continuous-time random walks for ordinary and equilibrium cases: Facilitation of diffusion, ergodicity breaking and ageing, Phys. Chem. Chem. Phys. 20, 20827 (2018).

[50] C. Godrèche and J. M. Luck, Statistics of the occupation time of renewal processes, J. Stat. Phys. 104, 489 (2001). 